\title{
Community Based Cancer Biomarker Identification from Gene Co-expression Network
}

\author{
Raihanul Bari Tanvir, Mona Maharjan, Ananda Mohan Mondal \\ School of Computing and Information Sciences \\ Florida International University \\ Miami, FL, USA \\ \{rtanv003, mmaha021, amondal\}@fiu.edu
}

\begin{abstract}
Finding the biomarkers of cancers and the analysis of cancerdriving genes that are involved in these biomarkers are essential for understanding the dynamics of cancer. Gene expression profiling has been widely used for cancer research, and its patterns, combined with statistical and computational techniques have been explored in many types of cancer. Genes having correlations in terms of expression may form complexes, pathways, or participate in regulatory and signaling circuits [1][3].
\end{abstract}

Clusters of genes in co-expression networks are commonly used as functional units. Gene co-expressed across multiple samples are more likely to correspond to functional groups. Analysis of gene co-expression networks of different types of cancer found that various cancers share common characteristics and functions and genes related to these characteristics were found in [4]-[6]. This work is based on the hypothesis established by previous works that the dense clusters or communities in the gene coexpression networks of cancer patients may represent functional units regarding cancer initiation and progression.

Gene co-expression networks were constructed from RNA-seq gene expression data of three cancers - Breast Invasive Carcinoma (BRCA), Colorectal Adenocarcinoma (COAD) and Glioblastoma Multiforme (GBM), using Pearson Correlation with a strict threshold. Then communities were found using seven renowned community detection algorithms. The permutation test was performed on these communities to find the ones that were conserved in all co-expression networks. Survival analysis based on the genes of conserved communities concludes that some of these could predict the survival risk of patients, which can be considered as the biomarkers of cancer.

Permission to make digital or hard copies of part or all of this work for personal or classroom use is granted without fee provided that copies are not made or distributed for profit or commercial advantage and that copies bear this notice and the full citation on the first page. Copyrights for third-party components of this work must be honored. For all other uses, contact the Owner/Author.

ACM-BCB '19, September 7-10, 2019, Niagara Falls, NY, USA

(C) 2019 Copyright is held by the owner/author(s).

ACM ISBN 978-1-4503-6666-3/19/09.

https://doi.org/10.1145/3307339.3343254
The discovered biomarker communities were also validated using survival analysis of two different cancers - Glioma (GBMLGG) and Ovarian Serous Cystadenocarcinoma (OV) - which were not used in finding the communities.

\section{KEYWORDS}

Cancer Biomarkers, Community Detection, Gene Co-expression Network.

\section{ACKNOWLEDGEMENT}

This research is funded by NSF CAREER award \#1651917 (transferred to \#1901628) to AMM.

\section{ACM Reference Format:}

Raihanul Bari Tanvir, Mona Maharjan and Ananda Mohan Mondal. 2019. Community Based Cancer Biomarker Identification from Gene Coexpression Network. In ACM BCB'19: The 10th ACM Conference on Bioinformatics, Computational Biology, and Health Informatics, September 7-10, New York, USA. ACM, New York, NY, USA, one page. https://doi.org/10.1145/3307339.3343254

\section{REFERENCES}

[1] Y. Huang et al., "Systematic discovery of functional modules and context-specific functional annotation of human genome," Bioinformatics, vol. 23, no. 13, pp. i222-i229, 2007.

[2] T. Ideker, O. Ozier, B. Schwikowski, and A. F. Siegel, "Discovering regulatory and signalling circuits in molecular interaction networks," Bioinformatics, vol. 18, no. suppl_1, pp. S233-S240, 2002.

[3] M. B. Eisen, P. T. Spellman, P. O. Brown, and D. Botstein, "Cluster analysis and display of genome-wide expression patterns," Proc. Natl. Acad. Sci., vol. 95, no. 25, pp. 14863-14868, 1998.

[4] H. K. Lee, A. K.-H. Hsu, J. Sajdak, J. Qin, and P. Pavlidis, "Coexpression analysis of human genes across many microarray data sets.," Genome Res., vol. 14 6, pp. 1085-1094, 2004.

[5] D. Hanahan and R. A. Weinberg, "Hallmarks of Cancer: The Next Generation," Cell, vol. 144, no. 5, pp. 646-674, 2011.

[6] Y. Yang, L. Han, Y. Yuan, J. Li, N. Hei, and H. Liang, "Gene coexpression network analysis reveals common system-level properties of prognostic genes across cancer types," Nat. Commun., vol. 5, p. 3231, 2014. 